\title{
1 Identification of a novel lineage bat SARS-related coronaviruses that use bat
}

\section{ACE2 receptor}

3 Hua Guo ${ }^{\text {a,b\# }}$, Ben $\mathrm{Hu}^{\text {a\# }}$, Hao-rui $\mathrm{Si}^{\mathrm{a}}{ }^{\mathrm{b} \#}$, Yan $\mathrm{Zhu}^{\mathrm{a}}$, Wei Zhang ${ }^{\mathrm{a}}$, Bei $\mathrm{Li}^{\mathrm{a}}$, Ang $\mathrm{Li}^{\mathrm{a}, \mathrm{b}}$, Rong

$4 \quad$ Geng $^{\mathrm{a}, \mathrm{b}}$, Hao-Feng Lin ${ }^{\mathrm{a}, \mathrm{b}}$, Xing-Lou Yang ${ }^{\mathrm{a}}$, Peng Zhou ${ }^{\mathrm{a}^{*}}$, Zheng-Li Shi ${ }^{\mathrm{a}^{*}}$

5

6 a. CAS Key Laboratory of Special Pathogens and Biosafety, Wuhan Institute of Virology,

7 Center for Biosafety Mega-Science, Chinese Academy of Sciences, Wuhan 430071, China.

8 b. University of Chinese Academy of Sciences, Beijing, 100049, China.

9

10 \#These authors contributed equally.

11 *To whom correspondence should be addressed: peng.zhou@wh.iov.cn;

12 zlshi@wh.iov.cn

13

\section{Author contributions}

15 Z-L.S and P.Z. conceived the study. X-L.Y organized sampling. B.H., H-R.S, B.L. 16 and Y.Z. performed viral genome sequencing and bioinformatics analysis. H.G.,W.Z.,

17 A.L. and R.G. performed protein expression and RBD-ACE2 binding assays. H.G.

18 and H-F.L. performed pseudovirus work. H.G, H.B, P.Z and Z-L.S wrote the paper

19 with input from all authors. 


\section{Abstract}

23 Severe respiratory disease coronavirus-2 (SARS-CoV-2) causes the most devastating

24 disease, COVID-19, of the recent century. One of the unsolved scientific questions

25 around SARS-CoV-2 is the animal origin of this virus. Bats and pangolins are

26 recognized as the most probable reservoir hosts that harbor the highly similar SARS-

27 CoV-2 related viruses (SARSr-CoV-2). Here, we report the identification of a novel

28 lineage of SARSr-CoVs, including RaTG15 and seven other viruses, from bats at the

29 same location where we found RaTG13 in 2015. Although RaTG15 and the related

30 viruses share $97.2 \%$ amino acid sequence identities to SARS-CoV-2 in the conserved

31 ORF1b region, but only show less than $77.6 \%$ to all known SARSr-CoVs in genome

32 level, thus forms a distinct lineage in the Sarbecovirus phylogenetic tree. We then

33 found that RaTG15 receptor binding domain (RBD) can bind to and use Rhinolophus

34 affinis bat ACE2 (RaACE2) but not human ACE2 as entry receptor, although which

35 contains a short deletion and has different key residues responsible for ACE2 binding.

36 In addition, we show that none of the known viruses in bat SARSr-CoV-2 lineage or

37 the novel lineage discovered so far use human ACE2 efficiently compared to SARSr-

$38 \mathrm{CoV}-2$ from pangolin or some of the SARSr-CoV-1 lineage viruses. Collectively, we

39 suggest more systematic and longitudinal work in bats to prevent future spillover

40 events caused by SARSr-CoVs or to better understand the origin of SARS-CoV-2.

41

42

43

44

45

Keywords: SARS-related coronavirus, Novel lineage, Bat, Reservoir host, ACE2, 
46

47

48

49

50

51

52

53

54

55

56

57

58

59

60

61

62

63

64

65

66

67

68

69

70

\section{Introduction}

SARS-CoV-2, a novel coronavirus that causes COVID-19 which was first identified in late 2019 [1], took just a few months to sweep the globe. As the largest pandemic in the past century in human history, it not only results in serious impact on human health but also leads to stagnation in economics, travel, education and many other societal functions globally.

The natural origin of SARS-CoV-2 is one of the unanswered scientific questions about the COVID-19 pandemic. It is generally believed that SARS-CoV-2 is transmitted from an animal reservoir host to human society through an or multiple intermediate hosts [2]. The discovery of SARS-CoV-2 related viruses (SARSr-CoV2), RaTG13 and Pangolin-CoV from horseshoe bats and pangolin respectively, shed light on the importance of these two groups as animal reservoirs of SARSr-CoV-2 viruses $[1,3,4]$. However, among the six critical residues of the receptor-binding domain (RBD) in spike to interact with human ACE2 receptor, RaTG13 only shares one with SARS-CoV-2 [5]. The RBD of RaTG13 has a lower binding affinity and usage efficiency with human ACE2 though sharing 96\% genome sequence identity to SARS-CoV-2 [6-8]. One of the viruses derived from Malayan pangolin (Manis javanica), Pangolin-CoV-GD, possesses six identical critical residues of RBD with SARS-CoV-2 and displays a similar binding affinity to human ACE2 compared with SARS-CoV-2, although it shares lower sequence identity to SARS-CoV-2 in genome compared with RaTG13 [4,7,8]. Another SARSr-CoV-2 detected from bat (Rhinolophus malayanus), RmYN02, contains a similar insertion at the S1/S2 cleavage site in the spike of SARS-CoV-2, but it has some deletions in the RBD and fails to bind with human ACE2 [9]. Besides, more SARSr-CoV-2 viral genome 
71 sequences from bats have been reported from Eastern China, Japan, and Southeast

72 Asian countries subsequently [10-13]. However, the progenitor virus that shares

$73>99 \%$ identical to SARS-CoV-2 was still undetermined.

Bats also carry SARSr-CoV with all the genetic building blocks of SARS-CoV-1, which jumped to human in 2002 [14]. Therefore, investigation of bat SARSr-CoVs is not only important for tracing the origin and immediate progenitor viruses of SARS-

CoV-2, but also critical for public health measures to prevent future outbreaks caused by this species of viruses. Here, we report the genome characterization and viral receptor analysis of a novel lineage of SARSr-CoVs in Tongguan town, Mojiang county, Yunnan province in China in 2015, the same location where we found bat RaTG13 in 2013 [1].

83

\section{Methods}

\section{Bat Sampling and Coronavirus Detection}

86 Sampling of bat was conducted in Mojiang county, Yunnan province at May 2015.

87 Bats were released after anal swabs sampling. Samples were aliquoted and stored at -

$8880^{\circ} \mathrm{C}$ until use. RNA was extracted using the High Pure Viral RNA Kit (Roche,

89 Basel, Switzerland). Partial RdRp was amplified using the SuperScript III OneStep

90 RT-PCR and Platinum Taq Enzyme kit (Invitrogen, Carlsbad, CA, USA) by family-

91 specific degenerate seminested PCR. The PCR products were gel purified and

92 sequenced with an ABI Prism 3700 DNA analyzer (Applied Biosystems, Foster City,

$93 \mathrm{CA})$. The sequences were blasted against the GenBank database.

94

\section{Genome Sequencing}


96 For SARSr-CoV positive RNA extractions, next-generation sequencing (NGS) was

97 performed using BGI MGISEQ 2000. NGS reads were first processed by Cutadapt

98 (v.1.18) to eliminate the possible contamination. Then the clean reads were assembled

99 into genomes using Geneious (v11.0.3) and MEGAHIT (v1.2.9). PCR and Sanger

100 sequencing were used to fill the genome gaps. To amplify the terminal ends, a

101 SMARTer RACE 5/3`kit (Takara) was used. Bat species identification was based on

102 the partial sequence of cytochrome c oxidase subunit I (COI) gene.

103

104

\section{Phylogenetic Analysis}

105 Routine sequence management and analysis were carried out using DNAStar.

106 Sequence alignments were created by ClustalW implemented in MEGA6 with default

107 parameters. Maximum Likelihood and Neighbour-joining phylogenetic trees were

108 generated using the Jukes-Cantor model with 1000 bootstrap replicates in the MEGA6

109 software package. Similarity plot analysis of the full-length genome sequences was

110 conducted by Simplot 3.5.1. The genome ID used in the analysis are MN996528 for

111 SARS-CoV-2, AY278488 for SARS-CoV-1, MN996532 for the bat SARSr-CoV

112 RaTG13, MG772933 for ZC45, MW251308 for RacCS203, LC556375 for Rc-o319,

113 KF367457 for WIV1, DQ022305 for HKU3-1, MT121216 for pangolin-CoV-GD

114 strain, MT072864.1 for pangolin-CoV-GX strain, EPI_ISL_412977 for bat SARSr-

115 CoV RmYN02, EPI_ISL_852604 for RshSTT182. The National Genomics Data

116 Center of China ID for the eight novel lineage SARSr-CoVs are:

117 GWHBAUM01000000- GWHBAUT01000000.

118

119 Expression Constructs, Protein Expression, and Purification 
120 Codon-optimized RBD genes from the following viruses were used (see above

121 genome accession number): SARS-CoV-2 (spike aa 330-583), SARS-CoV-1 (spike aa

122 317-569), RaTG13 (spike aa 330-583), pangolin-CoV-GD (spike aa326-579),

123 pangolin-CoV-GD (spike aa 330-583), RaTG15 (spike aa 317-566). They were

124 synthesized (Sangon Biotech, Shanghai, China) and placed into the expression vector

125 with an N-terminal signal peptide and an S-tag as described previously [15]. The

126 ectodomains of human ACE2 (aa 19-615, accession number: AB046569) and

127 R.affinis ACE2 (aa 19-615, accession number: MT394204) were amplified and

128 cloned into the same expression vector as above.

129

130 The RBD and ACE2 proteins used for the BLI binding assay were produced in HEK

$131293 \mathrm{~T} / 17$ cells. Cells were transiently transfected with expression plasmids using

132 Lipofectamine 3000 (Life Technologies), washed twice with D-Hanks solution $6 \mathrm{~h}$

133 post-transfection, and followed with culturing in fresh 293T FreeStyle expression

134 medium (Life Technologies) at $37^{\circ} \mathrm{C}$ in a humidified $5 \% \mathrm{CO} 2$ incubator. The

135 supernatant were harvested $48 \mathrm{~h}$ post-transfection and centrifuged at $4000 \times g$ for 10

$136 \min$ at $4^{\circ} \mathrm{C}$. Clarified supernatant were purified by S-tag agarose beads and eluted

137 with $3 \mathrm{M} \mathrm{MgCl}_{2}$. The purified protein was finally buffered with PBS and quantified

138 using Qubit 2 Fluorometer (Thermo Fisher Scientific), and stored at $-80{ }^{\circ} \mathrm{C}$ until use.

139

140 Bio-layer Interferometry Binding Assays

141 Binding assays between RBDs and ACE2 proteins were performed using the Octet

142 RED system (ForteBio, Menlo Park, CA, USA) in 96-well microplates at $30^{\circ} \mathrm{C}$ with

143 shaking at $1000 \mathrm{rpm}$ as described previously [15]. Briefly, the RBD was biotinylated

144 using EZ-Link NHS-LC-LC-Biotin (Thermo Fisher Scientific, Waltham, MA, USA). 
145 The Streptavidin Biosensors were activated for 200s prior to coupling with $50 \mu \mathrm{g} / \mathrm{mL}$

146 biotinylated RBD proteins for 600s. A baseline were collected in the kinetic buffer (1

$147 \mathrm{M} \mathrm{NaCl}, 0.1 \% \mathrm{BSA}, 0.02 \%$ Tween-20; $\mathrm{pH}$ 6.5) for 200s before immersing the sensors

148 in a 1:2 serial diluted ACE2 proteins for 900s and then dissociation in the same

149 kinetic buffer for another 900s. Data analysis from the ForteBio Octet RED

150 instrument includes reference subtraction. Inter-step correction and Y-alignment were

151 used to minimize tip-dependent variability. Curve fitting were performed in a 1:1

152 model using the Data Analysis Software v7.1 (ForteBio, Menlo Park, CA, USA). The

153 mean Kon, Koff values were determined with a global fit applied to all data. The

154 coefficient of determination $\left(\mathrm{R}^{\wedge} 2\right)$ for these interactions was close to 1.0.

155

156 Pseudovirus Entry Assays

157 Pseudotyped VSV $-\triangle \mathrm{G}$ particles were generated as previously described with minor

158 adjustments [16]. Briefly, HEK 293T/17 cells were seeded at 6-well-plate and

159 transfected with plasmids contain codon-optimized SARSr-CoV-2 spike at a 70\%

160 confluency using Lipofectamine 3000. At $6 \mathrm{~h}$ post-transfection, the medium was

161 replaced with fresh DMEM+10\%FBS medium. At $24 \mathrm{~h}$ after transfection, cells were

162 incubated with VSV-G-pseudotyped VSV $\triangle \mathrm{G} /$ Fluc at $37^{\circ} \mathrm{C}$ for $1 \mathrm{~h}$. Cells were

163 subsequently washed five times and supplied with fresh DMEM + 10\% FBS medium

$164+$ anti-VSV-G antibody (Kerafast). Cell-free supernatants were harvested at $24 \mathrm{~h}$ after

165 transduction, then centrifuged at $4000 \times g$ for $10 \mathrm{~min}$ at $4^{\circ} \mathrm{C}$. The virus particles were

166 used for infection directly. 
168 The 48-well-plate was treated with Poly-L-lysine solution (Sigma) before seeded

169 HEK293T/17 cells. Cells were transient transfected with equal amounts of human

170 ACE2, R.affinis ACE2 or empty vector plasmids at $70 \%$ confluency. At 24 h post-

171 transfection, the cells were incubated with same amounts of S-pseudotyped virions for

$1721 \mathrm{~h}$ at $37^{\circ} \mathrm{C}$, then washed twice with PBS solution, and supplemented with DMEM

173 containing 10\% FBS. Luciferase activity was determined using a GloMax

174 luminometer (Promega Biotech Co. Ltd., Beijing, China) $48 \mathrm{~h}$ after infection.

175 Infection experiments were performed independently in triplicate with three technical 176 replications each time.

179 Viral RNA of all VSV-spike pseudovirus particles were extracted from 200ul

180 supernatant using the High Pure Viral RNA Kit (Roche, Cat. No. 11858882001)

181 following the supplier's manual. Quantification of pseudovirus by real-time PCR was

182 performed using HiScript ${ }^{\circledR}$ II One Step qRT-PCR SYBR Green Kit (Vazyme, Cat.

183 No. Q221-01). The gene of VSV P protein were amplified and synthesized in vitro

184 using mMESSAGE mMACHINE® Kit (Life technologies, Cat. No. AM1344) to

185 serve as a standard. Viral copy numbers were calculated according to the standard

186 curve. Primers using for transcription in vitro were: VSV (P protein)-F1:

187 GTTCGTGAGTATCTCAAGTCCT, VSV (P protein)-R2-T7:

188 TAATACGACTCACTATAGGGAGAGCCTTGATTGTCTTCAATTTCTGG,

189 primers using for real-time PCR were described as previously [17].

190

191 Results

192 Identification of a novel lineage of SARSr-CoVs 
193 In tracing the origin of SARS-CoV-2 from bats, we identified RaTG13, which shares

$19496.2 \%$ genome identity to SARS-CoV-2 and is so far the closest genome [1].

195 Following the investigation, we identified eight SARSr-CoV sequences that share

$19693.5 \%$ sequence identity to SARS-CoV-2 in the 402-nt partial RdRp gene from bat

197 samples collected at the same place in 2015. Seven samples were from Rhinolophus

198 stheno, and the other one was from Rhinolophus affinis (Table S1). We thus

199 performed next-generation sequencing (NGS) for further analysis of these CoVs.

200 Whole genome sequences were obtained from all eight individual samples. The eight

201 SARSr-CoV genomes are almost identical, sharing more than $99.7 \%$ sequence

202 identity among each other. One strain designated RaTG15 was used as the

203 representative in the subsequent analysis.

204

205 In the seven conserved replicase domains used for coronavirus species classification,

206 RaTG15 is $95.3 \%$ or $92.5 \%$ identical to SARS-CoV-2 and SARS-CoV-1 respectively,

207 suggesting that it remains a member of the SARSr-CoV species in the Sarbecovirus

208 subgenus within Betacoronavirus genus, Coronaviridae family. Further, RaTG15 is

209 genetically close to SARS-CoV-2 in open reading frame $1 \mathrm{~b}$ (ORF1b). In the complete

210 ORF1b region, RaTG15 showed 84.6 89.0\% nucleotide identities and 95.6 97.3\%

211 amino acid sequence identities to bat SARSr-CoV-2 from wildlife in China and

212 Southeast Asia, which includes bat CoVs RaTG13 and RmYN02 from Yunnan, Rc-

213 o319 from Japan, RshSTT182 from Cambodia, RacCS203 from Thailand, as well as

214 two different strains of pangolin-CoVs (Table S2). It is also conceivable that RaTG15

215 clusteres with SARSr-CoV-2 in the phylogeny using full-length RdRp gene (Figure

216 S1A).

217 
218 In contrast, similarity plot analysis reveals that beyond ORF1b, RaTG15 is

219 remarkably distinct from both SARSr-CoV-2 and SARSr-CoV-1 in majority of the

220 genome (Figure 1A). It exhibits less than $80 \%$ nucleotide identities in ORF1a, M and

$221 \mathrm{~N}$ genes and lower than 70\% identities in S, ORF3, 6 and $7 \mathrm{a} / 7 \mathrm{~b}$ to all other SARSr-

222 CoVs (Table S2). Overall, the full genome of the SARSr-CoV RaTG15 show 74.4\%

223 sequence identity to SARS-CoV-1 and $77.6 \%$ to sequence identity to SARS-CoV-2.

224 Notably, RaTG15 show higher sequence identity to SARS-CoV-1 than to SARS-

$225 \mathrm{CoV}-2$ in the spike, E, M, N and ORF6 proteins. It also has almost equivalent

226 homology to any other known SARSr-CoVs from bat or pangolin CoVs (Table S2).

227 This mosaic profile suggests that this novel lineage viruses may be a results of

228 recombination of different SARSr-CoVs.

229

230 The result of phylogenetic analysis is in accordance with similarity plot. SARSr-CoVs

231 mainly consists of two sub-lineages, the SARSr-CoV-1 and SARSr-CoV-2 (Figure

232 1B). The latter one includes SARS-CoV-2 from pangolins and different Rhinolophus

233 bats species recently reported in a wide range of areas in Asia. In the full-length

234 genome tree and S gene tree, RaTG15 and the related viruses are distant from both of

235 the two existing sub-lineages, and forms a well-supported novel lineage with the

236 sarbecoviruses (Figure 1B and Figure S1B).

237

238 In silico analysis of receptor binding domain (RBDs) of SARSr-CoVs

239 We further examined the spike protein sequence of RaTG15 in comparison with other

240 SARSr-CoV-2. The receptor-binding domain (RBD) of the RaTG15 spike is highly

241 divergent from other sarbecoviruses, with $72.6 \%$ amino acid sequence identity to

242 SARS-CoV-2 and 68.6\%-73.3\% identities to related bat and pangolin CoVs. Unlike 
243 RmYN02 and RacCS203, the RaTG15 RBD does not contain the deletion

244 corresponding to aa 473-486 (deletion 2) of the SARS-CoV-2 spike which determines

245 ACE2 usage based on previous reports [18]. However, aligned with SARS-CoV-2 and

246 RaTG13, a short deletion is noted at the position corresponding to aa 444-447

247 (deletion 1). The location of this deletion is similar to the one in the spike of

248 RshSTT182, a SARS-CoV-2-related CoV identified in Rhinolophus shameli from

249 Cambodia. Within the receptor binding motif (RBM), four of the five amino acid

250 residues critical for binding of SARS-CoV-2 to the ACE2 receptor (486, 493, 494 and

251 501) are varied in RaTG15. Like most bat SARSr-CoVs, the polybasic (furin)

252 cleavage site is absent at the S1-S2 junction of RaTG15 (Figure 2).

253

254 Functional comparison of RBD from three lineages of SARSr-CoVs

255 The sequence analysis indicated that the RaTG15 virus possibly uses ACE2 as an

256 entry receptor, which was then experimentally confirmed by RBD-ACE2 binding

257 studies using purified recombinant proteins. RBD proteins from SARS-CoV-2,

258 SARS-CoV-1, RaTG13, pangolin-CoV-GD, pangolin-CoV-GX and RaTG15, as well

259 as ectodomains of human and R.affinis ACE2 proteins were used (Figure S2A). We

260 found that R.affinis derived RaTG13 and RaTG15 RBD proteins either show very

261 weak or have no binding affinity to human ACE2 (HuACE2). In contrast, RBD

262 proteins from the two pangolin SARSr-CoVs displayed much higher binding affinity

263 to HuACE2, only slightly weaker than SARS-CoV-2 RBD but still higher than

264 SARS-CoV-1 (Figure 3A-F and M). Furthermore, the binding affinity to HuACE2 of

265 pangolin-CoV-GX is slightly weaker than pangolin-CoV-GD. Next, we wanted to

266 find out whether bat CoVs RaTG13 and RaTG15 can use R.affinis ACE2 more

267 efficiently than huACE2. Detectable binding was observed between RaTG15 RBD 
268 and R.affinis ACE2 (RaACE2), though the affinity was still weaker than SARS-CoV-

2692 and pangolin-CoV-GD/GX to RaACE2. RaTG13 RBD showed a very weak binding

270 to RaACE2, same as to HuACE2 (Figure 3G-M and Figure S2B).

271

272 To exclude the possibility that the ACE2 binding of RBD may not represent the

273 functionality of the full-length S protein, we also constructed a VSV-based

274 pseudovirus using previously published method [16]. We produced a list of SARSr-

275 CoVs pseudoviruses, or MERS-CoV pseudovirus as a negative control. HEK293T/17

276 cells overexpression HuACE2, RaACE2 or empty vector were infected with VSV-

277 based pseudoviruses, and the infection efficiency were determined $48 \mathrm{~h}$ after

278 infection. Consistent with the RBD-ACE2 protein binding assays, HuACE2 mediated

279 entry of all SARSr-CoVs except the RaTG15, whereas the R.affinis ACE2 supported

280 all SARSr-CoVs entry. Notably, RaTG13 pseudovirus infection of HuACE2 or

281 RaACE2-expression cells was minimal, if it is positive, compared to other groups. As

282 control, MERS-CoV pseudovirus failed to infect ACE2-expression cells, confirming

283 ACE2-independent infectivity of VSV backbone (Figure S3). Collectively, none of

284 the SARSr-CoV-2 lineage or the novel lineage virus from bats could efficiently bind

285 to HuACE2 [10,11], and it appears that whether there is deletion at RBD region

286 greatly affecting the binding capacity (Figure $3 \mathrm{~N}$ ). These results suggest that without

287 further adaptation, there is a limited zoonotic potential for bat-derived RaTG13,

288 RaTG15 and perhaps other SARSr-CoV-2 lineage or the novel lineage viruses. In

289 contrast, there is a high spillover potential of pangolin-CoV in the context of cell

290 receptor usage.

291

292 Discussion 
293 Overall, we report the discovery of a novel lineage of SARSr-CoVs from bats that are

294 closely related to SARS-CoV-2 in the RdRp region, but genetically distant to any

295 known SARSr-CoVs at genome level. Although several SARS-CoV-2 related

296 coronaviruses have been detected from wildlife, none of them shared $>99 \%$

297 genetically identical to SARS-CoV-2 at the genome level. Recombination events

298 happen commonly in coronaviruses and can be referred to as potential origin of the

299 progenitor of SARS-CoV-1, as SARSr-CoVs discoved in a bat colony carried all the

300 genomic fragments of SARS-CoV-1 $[14,19]$. The high sequence similarity to SARS-

$301 \mathrm{CoV}-2$ in some genomic regions detected from different wildlife species implies the

302 recombination may happen during the virus evolution in cross-species or inter-species

303 transmission. The new lineage virus we reported in this study showed weak binding

304 affinity to bat but not human ACE2 though possess one deletion in the RBD of the

305 spike which is different from the previously reported SARSr-CoVs in bat (Figure 2).

306 These results suggested the SARSr-CoVs we discovered from bat now may be just the

307 tip of the iceberg. These viruses may have experienced selection or recombination

308 events in the animal hosts and render viral adaption to a new host then spread to the

309 new species before they jumped into human society. So surveillance to this new

310 lineage virus should be conducted to prevent future outbreaks, as viruses from the

311 other two lineages of SARSr-CoV caused SARS and COVID-19, respectively $[1,20]$.

312 Furthermore, none of the bat SARSr-CoV-2 lineage or the novel lineage viruses

313 discovered so far could be isolated, or be capable of efficiently using human ACE2,

314 thus pose little spillover potential to human without future adaptation [21]. In

315 comparison, the ACE2 usage virus in bat SARSr-CoV-1 related lineage appears to be

316 more dangerous in the context of cross-species transmission, which has been

317 demonstrated in animal studies [22,23]. 
319 The closest bat CoV to SARS-CoV-2 at this stage, RaTG13 only showed very weak

320 binding affinity to HuACE2. Albeit there is a speculation claiming the possible

321 leaking of RaTG13 from lab that caused SARS-CoV-2, the experiment evidence

322 cannot support it. In contrast, the pangolin-CoV shows strong binding capacity to

323 human or bat ACE2, posing high cross-species potential to human or other species. In

324 the context of SARS-CoV-2 animal origin, there could either be a bat SARSr-CoV

325 closer than RaTG13 that is capable of using HuACE2, or be a pangolin-CoV that

326 obtained higher genome similarity other than spike gene. In future, more systematic

327 and longitudinal sampling of bats, pangolins or other possible intermediate animals is

328 required to better understand the origin of SARS-CoV-2.

329

330 Acknowledgements

331 We thank Yun-Zhi Zhang, Ji-Hua Zhou from Yunnan CDC for helping with bat

332 sampling. We also would like to thank Dr. Ding Gao in the WIV Core Facility and

333 Technical Support for his help in Octet RED technology. The work was jointly

334 supported by the Strategic Priority Research Program of the Chinese Academy of

335 Sciences (XDB29010101, to Z-L.S) and China National Science Foundation for

336 Excellent Scholars (81290341 to Z-LS, 81822028 to P.Z.).

337

338 Declaration of Interests

339 The authors declare no competing interests.

\section{References}


342 1. Zhou P, Yang X-L, Wang X-G, et al. A pneumonia outbreak associated with

343

344

345

346

347

348

349

350

351

352

353

354

355

356

357

358

359

360

361

362

363

364

365

366

367

368

369

370

371

372

373

374

375

376

377

378

379

380

381

382

383

384

385

386

387

388

389 a new coronavirus of probable bat origin. Nature. 2020 2020/03/01;579(7798):270-273.

2. Zhou P, Shi ZL. SARS-CoV-2 spillover events. Science. 2021 Jan 8;371(6525):120-122.

3. Xiao K, Zhai J, Feng Y, et al. Isolation of SARS-CoV-2-related coronavirus from Malayan pangolins. Nature. 2020 2020/07/01;583(7815):286-289.

4. Lam TT-Y, Jia N, Zhang Y-W, et al. Identifying SARS-CoV-2-related coronaviruses in Malayan pangolins. Nature. 2020 2020/07/01;583(7815):282-285.

5. Hu B, Guo H, Zhou P, et al. Characteristics of SARS-CoV-2 and COVID-19. Nature Reviews Microbiology. 2021 2021/03/01;19(3):141-154.

6. Shang J, Ye G, Shi K, et al. Structural basis of receptor recognition by SARSCoV-2. Nature. 2020 2020/05/01;581(7807):221-224.

7. Wrobel AG, Benton DJ, Xu P, et al. Structure and binding properties of Pangolin-CoV spike glycoprotein inform the evolution of SARS-CoV-2. Nature communications. 2021 2021/02/05;12(1):837.

8. Starr TN, Greaney AJ, Hilton SK, et al. Deep Mutational Scanning of SARSCoV-2 Receptor Binding Domain Reveals Constraints on Folding and ACE2 Binding. Cell. 2020 Sep 3;182(5):1295-1310 e20.

9. Zhou H, Chen X, Hu T, et al. A Novel Bat Coronavirus Closely Related to SARS-CoV-2 Contains Natural Insertions at the S1/S2 Cleavage Site of the Spike Protein. Curr Biol. 2020;30(11):2196-2203.e3.

10. Murakami S, Kitamura T, Suzuki J, et al. Detection and Characterization of Bat Sarbecovirus Phylogenetically Related to SARS-CoV-2, Japan.

Emerging Infectious Disease journal. 2020;26(12):3025.

11. Wacharapluesadee S, Tan CW, Maneeorn P, et al. Evidence for SARS-CoV-2 related coronaviruses circulating in bats and pangolins in Southeast Asia. Nature communications. 2021 2021/02/09;12(1):972.

12. Hu D, Zhu C, Ai L, et al. Genomic characterization and infectivity of a novel SARS-like coronavirus in Chinese bats. Emerg Microbes Infect. 2018 Sep 12;7(1):154.

13. Hul V, Delaune D, Karlsson EA, et al. A novel SARS-CoV-2 related coronavirus in bats from Cambodia. bioRxiv. 2021:2021.01.26.428212.

14. Hu B, Zeng LP, Yang XL, et al. Discovery of a rich gene pool of bat SARSrelated coronaviruses provides new insights into the origin of SARS coronavirus. Plos Pathog. 2017 Nov;13(11):e1006698.

15. Guo H, Hu B-J, Yang X-L, et al. Evolutionary Arms Race between Virus and Host Drives Genetic Diversity in Bat Severe Acute Respiratory SyndromeRelated Coronavirus Spike Genes. J Virol. 2020;94(20):e00902-20.

16. Johnson MC, Lyddon TD, Suarez R, et al. Optimized Pseudotyping Conditions for the SARS-COV-2 Spike Glycoprotein. J Virol. 2020;94(21):e01062-20.

17. Li Q, Wu J, Nie J, et al. The Impact of Mutations in SARS-CoV-2 Spike on Viral Infectivity and Antigenicity. Cell. 2020 Sep 3;182(5):1284-1294 e9.

18. Ren W, Qu X, Li W, et al. Difference in receptor usage between severe acute respiratory syndrome (SARS) coronavirus and SARS-like coronavirus of bat origin. J Virol. 2008 Feb;82(4):1899-907. 
19. Cui J, Li F, Shi ZL. Origin and evolution of pathogenic coronaviruses. Nature reviews Microbiology. 2019 Mar;17(3):181-192.

20. Drosten C, Gunther S, Preiser W, et al. Identification of a novel coronavirus in patients with severe acute respiratory syndrome. $\mathrm{N}$ Engl J Med. 2003 May 15;348(20):1967-76.

21. Yang XL, Hu B, Wang B, et al. Isolation and Characterization of a Novel Bat Coronavirus Closely Related to the Direct Progenitor of Severe Acute Respiratory Syndrome Coronavirus. J Virol. 2015 Dec 30;90(6):3253-6.

22. Menachery VD, Yount BL, Jr., Debbink K, et al. A SARS-like cluster of circulating bat coronaviruses shows potential for human emergence. Nat Med. 2015 Dec;21(12):1508-13.

23. Menachery VD, Yount BL, Jr., Sims AC, et al. SARS-like WIV1-CoV poised for human emergence. Proc Natl Acad Sci U S A. 2016 Mar 15;113(11):3048-53.

\section{Figure legends}

Figure 1. Discovery of a novel lineage of bat SARSr-CoVs. (A) Similarity plot analysis based on the full-length genome sequence of bat SARSr-CoV RaTG15. Fulllength genome sequences of SARS-CoV-1, SARS-CoV-2, bat and pangolin CoVs related to SARS-CoV-2 were used as reference sequences. The analysis was performed with the Kimura model, a window size of 1500 base pairs and a step size of 150 base pairs. (B) Phylogenetic tree based on complete genome sequences of betacoronaviruses. The trees were constructed by the Neighbour-joining method using the Jukes-Cantor model with bootstrap values determined by 1000 replicates.

Bootstraps $>50 \%$ are shown. The scale bars represent 0.1 substitutions per nucleotide position. The novel SARSr-CoVs characterized in this study are shown in bold. Ra, Rhinolophus affinis; Rst, Rhinolophus stheno; Rsh, Rhinolophus shameli; Rs, Rhinolophus sinicus; Rac, Rhinolophus acuminatus; Rm, Rhinolophus malayanus; Rc, Rhinolophus cornutus; MHV, murine hepatitis virus. 
421 Figure 2. Comparison of receptor-binding domain (RBDs) of SARSr-CoVs. The

422 RBM is shown in pink and the five key residues that contact ACE2 directly are

423 highlighted in green. Comparison of the five critical residues of these SARSr-CoVs

424 are listed in the table. Two deletions in the RBM, aa 444-447 (deletion 1) and aa 473-

425486 (deletion 2) are indicated by red boxes. GenBank or GISAID entries for each

426 virus can be found in Methods.

427

428

430

431

432

433

434

435

436

437

438

440

441

442

443

444 Figure S1. Phylogenetic tree base on the complete $\mathrm{S}$ gene sequences (A) or

445 complete RdRp gene sequences (B) of betacoronaviruses. The trees were

Figure 3. Binding affinity of SARSr-CoV RBDs to ACE2 from human and

R.affinis bat. (A-F) Binding of different RBD proteins to human ACE2. (G-L)

Binding of different RBD proteins to R.affinis ACE2. (M) Comparison of dissociation constants (KD) between different RBD to human and R.affinis ACE2. Relative binding is analyzed by comparing with SARS-CoV-2 RBD to human ACE2. (N)

Summary of the binding efficiency of different RBD to human or bat ACE2. Y, yes;

ND, not determined. Evidences for WIV16-CoV, Rc-o0319, RmYN02 and RacCS213

were from previous reports $[10,11,21]$. The presence of deletion in RBM (related to

Figure 2) is indicated. Binding assay of human or R.affinis ACE2 to different RBD

proteins was measured by Bio-layer interferometry. The parameters of KD value (M),

Kon (1/M.s), Koff (1/s) are shown on the upper right side of the picture. Different

RBD proteins were immobilized on the sensors and tested for affinity with graded

concentrations of human or $R$. affinis ACE2s. The Y-axis shows the real-time binding

response. Values reported representing the global fit to all data. The coefficient of

determination $\left(\mathrm{R}^{\wedge} 2\right)$ for these interactions was close to 1.0 (Figure $\mathrm{S} 2 \mathrm{~B}$ ). 
446 constructed by the Maximum-likelihood method using the Jukes-Cantor model with

447 bootstrap values determined by 1000 replicates. Bootstraps $>50 \%$ are shown. The

448 scale bars represent 0.1 and 0.05 substitutions per nucleotide position, respectively.

449 The novel SARSr-CoVs characterized in this study are shown in bold. Ra,

450 Rhinolophus affinis; Rst, Rhinolophus stheno; Rsh, Rhinolophus shameli; Rs,

451 Rhinolophus sinicus; Rac, Rhinolophus acuminatus; Rm, Rhinolophus malayanus; Rc,

452 Rhinolophus cornutus; MHV, murine hepatitis virus.

453

454

Figure S2. Binding affinity of SARSr-CoVs RBD proteins to ACE2 from human

and R.affinis. (A) The purity of different CoV-RBD and ACE2 proteins used for

binding assay were analyzed by SDS-PAGE. (B) Binding assay of human or R.affinis

457 ACE2 to different RBD proteins measured by Bio-layer interferometry, Related to

458 Figure 3. The Y-axis shows the real-time binding response. Values reported

459 representing the global fit to all data. The coefficient of determination $\left(\mathrm{R}^{\wedge} 2\right)$ for these

460 interactions was shown on the upper right.

461

462

Figure S3. Infectivity analysis of SARSr-CoV spike VSV-pseudoviruses in

463 human and R.affinis ACE2 expression cells. HEK293T/17 cells expression

464 human/R.affinis ACE2 were infected with SARS-CoV-2 and SARSr-CoV spike-

465 pseudotyped viruses. The infected cell lysis was analyzed by measuring luciferase

466 activities. All results were performed in triplicate from three independent

467 experiments. Error bars indicate mean \pm SEM. Statistical significance was tested by

468 one-way ANOVA with Dunnett posttest. (A) SARS-CoV-2, (B) RaTG15, (C)

469 pangolin-CoV-GD, (D) pangolin-CoV-GD, (E) RaTG13, (F) SARS-CoV-1, (G)

470 MERS-CoV. (H) Genome copies of VSV-CoV-S pseudotyped particles. Viral copy 
471 numbers were calculated according to the standard curve of VSV P protein gene. A

472 representative result is shown. (I) ACE2 expression was detected using mouse anti-S-

473 tag monoclonal antibody followed by HRP-labelled goat anti-mouse IgG antibody. $\beta$ -

474 actin was detected with mouse anti- $\beta$-action monoclonal antibody by HRP-labelled

475 goat anti-mouse $\operatorname{IgG}$ antibody.

476 


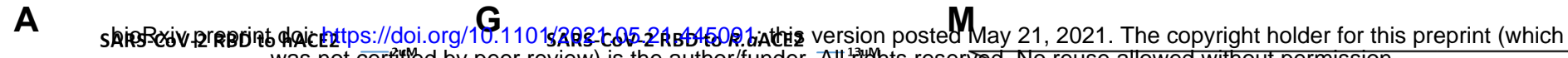

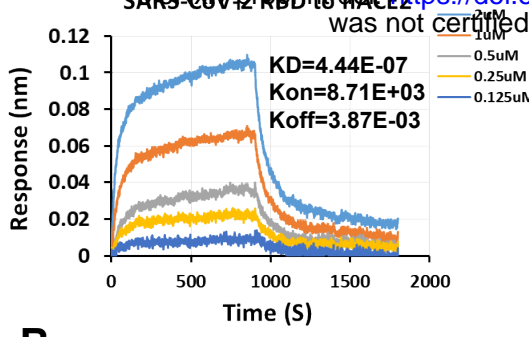

B

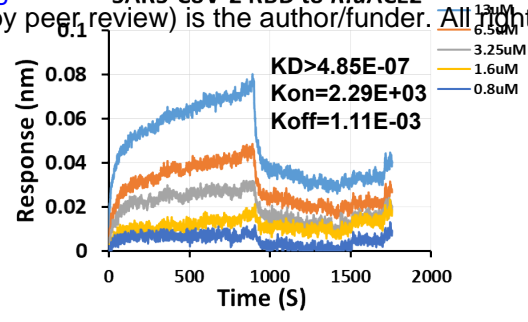

H

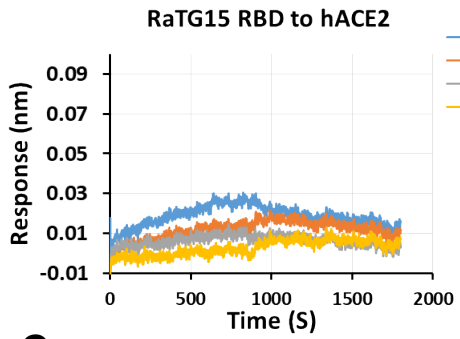

C
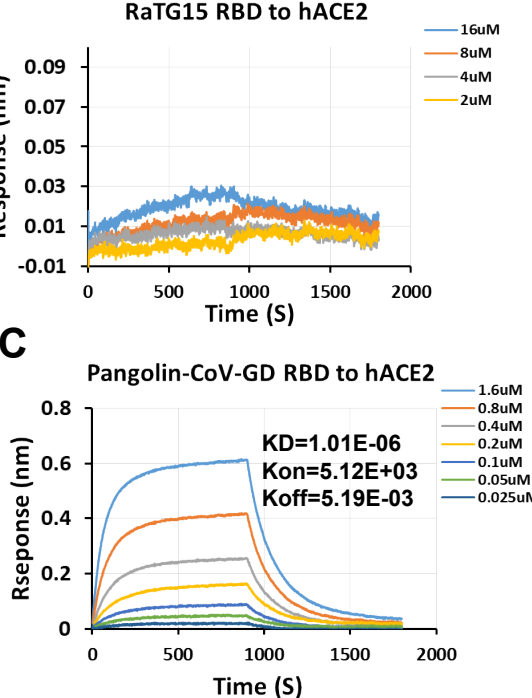

D
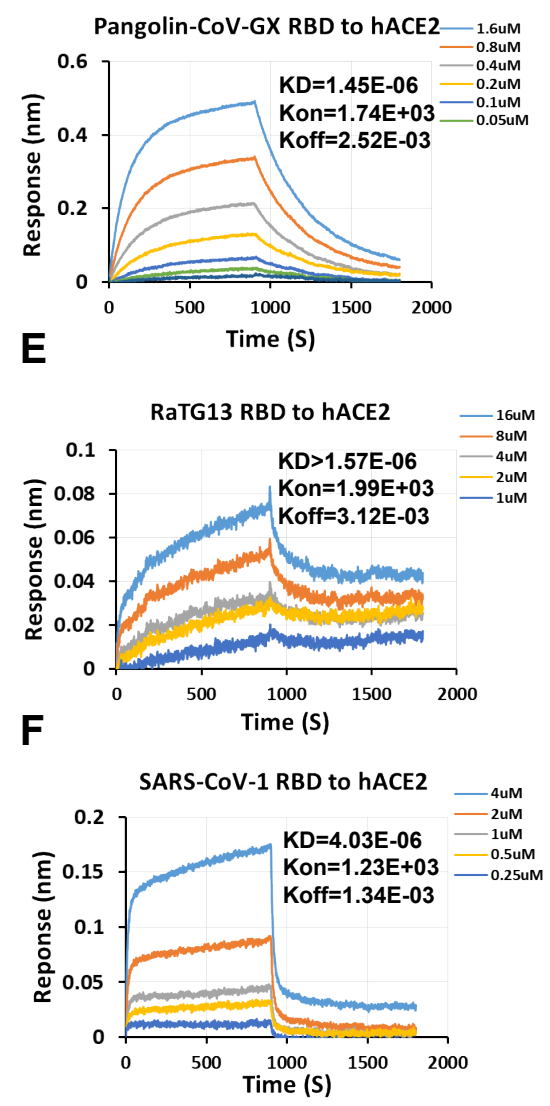

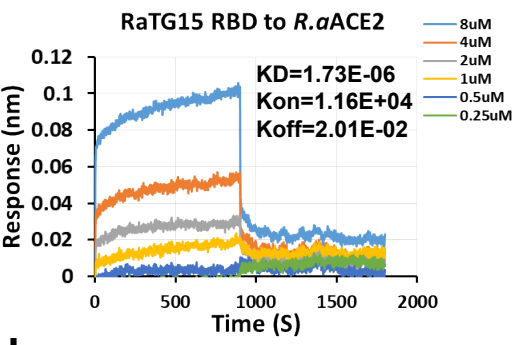

I

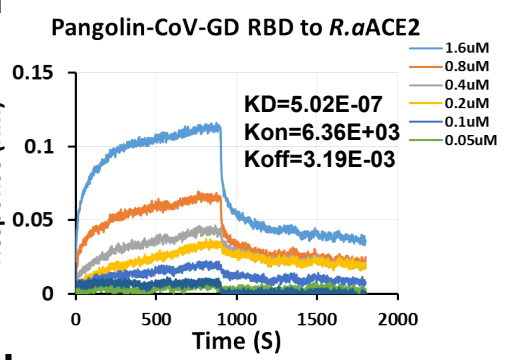

J
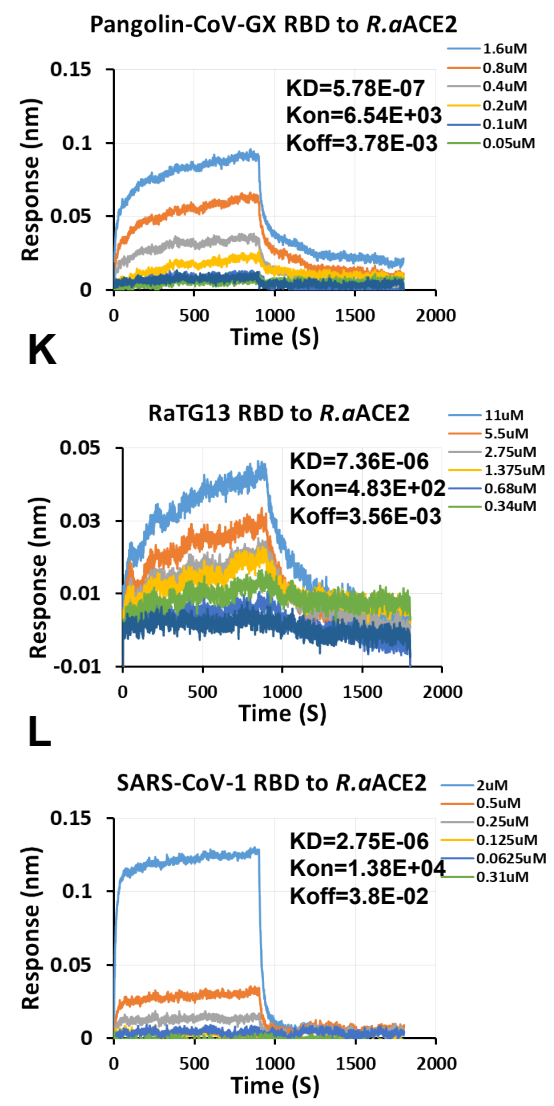

\begin{tabular}{|c|c|c|}
\hline $\begin{array}{l}\text { ACE2 } \\
\text { ACE reuse allowec }\end{array}$ & $\begin{array}{l}\text { thout permission. } \\
\text { Human }\end{array}$ & R.affinis \\
\hline SARS-CoV-2 & 1.00 & $>1.09$ \\
\hline RaTG15 & Not detectable & 3.90 \\
\hline Pangolin-CoV-GD & 2.28 & 1.13 \\
\hline Pangolin-CoV-GX & 3.26 & 1.30 \\
\hline RaTG13 & Low affinity, $>3.53$ & 16.57 \\
\hline SARS-CoV-1 & 9.07 & 6.19 \\
\hline
\end{tabular}

$\mathbf{N}$

\begin{tabular}{|c|c|c|c|c|c|}
\hline & & Human ACE2 & Bat ACE2 & Deletion 1 & Deletion 2 \\
\hline \multirow{3}{*}{$\begin{array}{l}\text { SARS-CoV-1 } \\
\text { related lineage }\end{array}$} & SARS-CoV-1 & $v$ & v & & \\
\hline & WIV16-CoV & $v$ & $\mathbf{v}$ & & \\
\hline & Rp3-CoV & $x$ & $x$ & $Y$ & $Y$ \\
\hline \multirow{7}{*}{$\begin{array}{l}\text { SARS-CoV-2 } \\
\text { related lineage }\end{array}$} & SARS-CoV-2 & v & v & & \\
\hline & RaTG13 & weak & weak & & \\
\hline & Rc-00319 & $x$ & v & & $\mathrm{Y}$, partial \\
\hline & RmYN02 & $x$ & ND & $Y$ & $Y$ \\
\hline & RacCS213 & $x$ & ND & $Y$ & $Y$ \\
\hline & Pangolin-CoV-GX & v & v & & \\
\hline & Pangolin-CoV-GD & v & v & & \\
\hline Novel lineage & RaTG15 & $x$ & v & $Y$ & \\
\hline
\end{tabular}

